\title{
Design and Benchtop Validation of a Powered Knee-Ankle Prosthesis with High-Torque, Low-Impedance Actuators
}

\author{
Toby Elery, Siavash Rezazadeh, Christopher Nesler, Jack Doan, Hanqi Zhu, Robert D. \\ Gregg \\ Departments of Bioengineering, Mechanical Engineering, and Electrical and Computer \\ Engineering, University of Texas at Dallas, Richardson, TX 75080 USA
}

Robert D. Gregg: rgregg@ieee.org

\begin{abstract}
This paper describes the design of a powered knee- and-ankle transfemoral prosthetic leg, which implements high torque density actuators with low-reduction transmissions. The low reduction of the transmission coupled with a high-torque and low-speed motor creates an actuator with low mechanical impedance and high backdrivability. This style of actuation presents several possible benefits over modern actuation styles implemented in emerging robotic prosthetic legs. Such benefits include free-swinging knee motion, compliance with the ground, negligible unmodeled actuator dynamics, and greater potential for power regeneration. Benchtop validation experiments were conducted to verify some of these benefits. Backdrive and free-swinging knee tests confirm that both joints can be backdriven by small torques $(\sim 3 \mathrm{Nm})$. Bandwidth tests reveal that the actuator is capable of achieving frequencies required for walking and running. Lastly, open-loop impedance control tests prove that the intrinsic impedance and unmodeled dynamics of the actuator are sufficiently small to control joint impedance without torque feedback.
\end{abstract}

\section{Introduction}

Ambulation using a passive prosthesis after the amputation of a lower limb results in a gait that is slower, less stable, and less energy efficient than able-bodied locomotion [1], [2]. Passive prostheses aim to alleviate the effects of amputation using mechanisms such as springs, cams, and dampers to mimic normative gait patterns. However, passive prostheses are limited in functionality due to the fact that such mechanisms can only dissipate energy that the user introduces. Although passive devices restore some functionality, amputees are typically left with an asymmetric gait [3]. Passive prostheses are also limited in their functionality across tasks. For example, many passive devices aim to mimic normal walking conditions. However, this does not address tasks such as sit-to-stand or stair ascent/descent. Semi-active prostheses, such as the Ottobock C-Leg, aim to resolve this by utilizing microprocessors to control the damping of joints with the use of small actuators that vary hydraulic valves during the user's gait [4], [5]. This approach allows for a single product to be easily adaptable to a variety of subjects, environments, and tasks. However, since this type of system only actively controls the damping at joints, it is an energy dissipating device, 
therefore not injecting any energy into the user's gait. By actively injecting energy into the user's gait, powered prostheses have the capability to restore mobility and quality of life to those who live with the loss of a limb.

In the last decade, a great amount of research has gone into the design and control of powered prosthetic limbs [6], [7]. Many powered prosthetic devices have emerged from this research, several of which implement non-backdrivable actuators, consisting of high-speed, low-torque motors with high-ratio transmissions, such as ball screws or multiple gear stages [4], [6], [8]-[16]. In the past few years, legged robots such as the quadruped MIT Cheetah [17], biped ATRIAS [18], and others [19] have embraced high-torque motors with low-ratio or no transmissions. Inspired by this approach, exoskeletons in the field of rehabilitation robotics have recently implemented high-torque motors in combination with low-ratio transmissions, such as the University of Texas at Dallas powered knee-ankle orthosis [20].

High-torque, low-reduction-ratio actuators can have several benefits for control and efficiency of robotic legs. The lower mechanical impedance (inertias and frictional losses) of these actuators minimizes the effect of unmodeled dynamics, which in turn helps simplify an otherwise complex control problem, increasing robustness, and forcing the system to behave closer to an ideal model. Force control in these actuators can be comparable to series elastic actuators without their design and manufacturing complexities. Other benefits include passive knee-swing motion, potential for power regeneration, and compliance with the ground through impedance control. A free-swinging knee joint allows for a more natural gait, while reducing the power requirements of the actuator during swing phase. Power regeneration phases of negative work on the leg, including swing knee extension, can lead to longer periods of untethered operation, which is critical for robotic legs. Lastly, compliance with the ground provides a smoother touchdown impact, which can in turn improve efficiency of the system and comfort for the user.

In the process of designing low-impedance actuators, transmission design is a critical problem. Single-stage planetary transmissions are extremely efficient and have less intrinsic impedance than multi-stage transmissions, but they are typically limited to ratios below 10:1. Therefore, efficient single-stage transmissions usually require a customized motor design such as [17] to achieve the high output torques required during legged locomotion. Other transmission choices used in robotic legs such as harmonic and cycloid gear drives exhibit other problems such as efficiency and manufacturing complexities, respectively. To overcome these shortcomings, we propose using a single-stage stepped-planet compound planetary gear transmission (SPC-PGT) [21] coupled with a high torque-density motor. As we will show, the resulting actuator has low mechanical impedance and high backdrivability. In addition, this style of transmission offers a higher range of reduction ratios while maintaining efficiency and simplifying manufacturing compared to previously mentioned transmissions.

The mechatronic design of the powered prosthetic leg is presented in Section II, including the motor, transmission, electrical system, and structure of both joints. Section III presents a series of benchtop experiments that characterize the velocity, torque, position tracking, and 
backdrive capabilities of the actuators. Section IV reflects upon the work presented and discusses the direction of our future work.

\section{Design}

\section{A. Design Overview}

We derive the requirements for the knee and ankle actuators from the necessary torque, velocity, position, and power requirements for level ground, stair ascent, and stair descent ambulation [22], [23], shown in Table I. Figure 1 presents the needed power throughout the gait cycle, including the power required by the electronics system. Lastly, a self-imposed requirement of an adjustable shank length allows for a larger population of potential user's. Structural components of both actuators were optimized using the finite element analysis software, ANSYS, to ensure structural integrity against impact and loading conditions of a $113.4 \mathrm{~kg}$ (250 lbs) user during level ground walking and stair ambulation. Most machined components were made of 7075-T6 aluminum, with a few shafts, gears, and bearings made of stainless steel. The assembled leg, shown in Figure 2 weighs approximately $6.05 \mathrm{~kg}$. In an effort to reduce weight, components that are under minimal loading conditions were 3D printed in ABS plastic. The leg is powered using a DC power supply, N8736, Keysight Technologies, California, USA.

\section{B. Motor and Driver}

High-torque motors typically used in industrial settings have large masses and volumes due to their robust housings and heat sinks. In addition, these motors are typically fixed in place, leading to minimal consideration of weight in their design. However, for implementation into a powered prosthetic leg, it was necessary for us to select a motor with high torque density, to ensure that our actuator could produce the required torque while remaining as light as possible. To this end, we selected the ILM $85 \times 26$ motor kit, Robodrive, Germany. This frameless, brushless DC motor kit allowed for the design of a custom housing that can withstand loading conditions and dissipate heat, while reducing the weight compared to industrial motor assemblies. This motor has a manufacturer rated torque of $2.6 \mathrm{Nm}$, peak torque of $8.3 \mathrm{Nm}$, and velocity up to $1500 \mathrm{rpm}$. Additionally, it is rated at $410 \mathrm{~W}, 48 \mathrm{~V}$, and $11 \mathrm{~A}$. The motor is driven by a 25/100 Solo Gold Twitter motor driver (Elmo Motion Control, Petah Tikva, Israel), which has a rated current of $17.6 \mathrm{~A}$ and a peak current of 35.2 A. Additionally, the driver's mass of $22.2 \mathrm{~g}$ is ideal for minimizing overall actuator mass.

\section{Transmission}

In order to increase the torque and reduce the speed of the motor to fit within the desired torque/velocity range, we designed a custom single-stage stepped-planet compound planetary gear transmission (SPC-PGT) with a 22:1 reduction. The SPC-PGT consists of one sun gear, one ring gear, and six planet gears procured from SDP/SI, New York, USA. Traditional planetary gear transmissions have only three planet gears, which mesh between the sun and ring gears. However, the SPC-PGT used here calls for three sun-planet gears and three ring-planet gears. Each sun-planet gear is coaxially fixed in relation to its corresponding ring-planet gear through a keyed shaft. The sun-planet gears mesh with the sun gear, radially located $120^{\circ}$ apart from each other. Similarly, the ring-planet gears are 
meshed with the ring gear, and are also radially located $120^{\circ}$ apart from each other. The shafts of the planet gears are held on either side by what is commonly referred to as a planet carrier. The transmission assembly can be seen in Figure 3.

Although planetary gear transmissions have multiple input-to-output configurations, the presented gearbox uses the sun gear as the input and the planetary carrier as the output to achieve the maximum ratio possible given a specific gear set. A traditional single-stage planetary gear transmission with the same input to output configuration has a reduction ratio found by $\tau_{m} / \tau_{j}=\left(D_{r}+D_{S}\right) /\left(D_{S}\right)$, whereas the reduction ratio of the single-stage SPC-PGT is found by $\tau_{m} / \tau_{j}=1+\left(D_{I} D_{\mathrm{sp}}\right) /\left(D_{S} D_{\mathrm{rp}}\right)$, where $\tau_{m}$ and $\tau_{j}$ are the motor and joint torque, respectively, and $D_{s}, D_{\mathrm{sp}}, D_{\mathrm{rp}}$, and $D_{r}$ are the sun, sun-planet, ring-planet, and ring gear diameter, respectively. Due to geometric constraints of a traditional planetary gear transmission, reduction ratios are typically limited to 10:1. However, the SPC-PGT can easily achieve higher reduction ratios in the same geometric volume. Although the presented design differs from a traditional single-stage planetary gear transmission, the number of gears meshed together is the exact same, thus increasing the obtainable reduction ratio while maintaining efficiency [24]. Coupled to the high-torque motor, this transmission provides a continuous torque of $57.2 \mathrm{Nm}$ and a peak torque of $182.6 \mathrm{Nm}$, demonstrating a larger scale application of a SPC-PGT transmission compared to the jumping robot in [25].

\section{Sensors and Electrical System}

Sensor feedback is critical for both the control and safety features of the device. Each actuator has one Optical Quadrature Encoder with 4096 cycles per revolution, US Digital, Washington, USA. Fixed to the motor shaft, the encoder sends motor position data to the motor driver and system controller. Once at the controller, this data is multiplied by the transmission reduction ratio for position and velocity feedback. Although only one encoder was used per actuator, the leg's design allows for a second encoder to be used at each actuator output, allowing direct joint measurements for position and velocity feedback. For this reason, some renderings show two encoders per actuator. Additionally, both motors contain two Pt1000 thermistors embedded in the stator. These monitor the internal temperature of the stator to ensure that the motor is not damaged during use. A M3564F 6axis load cell, Sunrise Instruments, Nanning, China, located below the ankle joint axis, provides force and moment information usable for ground detection and reaction forces during gait. It is capable of reading $2500 \mathrm{~N} / 200 \mathrm{Nm}$ along the $\mathrm{x}$ and $\mathrm{y}$ axes and $5000 \mathrm{~N} / 100$ $\mathrm{Nm}$ along the $\mathrm{z}$ axis.

These sensors interface with the system's microcontroller, a myRIO, National Instruments, Texas, USA. The controllers presented in Section III are implemented in the National Instruments LabVIEW software environment and then imported onto the myRIO. Figure 4 displays a systemic view of the described electrical system.

\section{E. Knee Mechanical Structure}

Although the physiological knee is a polycentric joint, it is often modeled as a single axis joint due to the minimal benefit gained from such an increase in design complexity [26], [27]. Therefore, the presented knee actuator shown in Figure 5 is designed as a simple hinge, 
which includes an upper and lower hinge piece. The upper hinge attaches to the socket on the user's residual limb via a pyramid adapter. The lower hinge is rigidly attached to the gearbox output, thus acting as the actuator output. Components of the actuator, such as the motor and transmission, are attached to the upper hinge, instead of the lower hinge, to minimize cable movement during gait. This design keeps the motor, transmission, and knee joint coaxial, which avoids the need for additional material/components to transfer motion from the motor axis to the knee joint.

This actuator is designed to allow simple changes to adjustable components so that the prosthesis may be configured for different use cases (i.e., modified range of motion and shank length). This is accomplished through the use of swappable hard stops and modular actuators separated by a pylon. Knee motion is constrained by bumpers that are 3D printed using a compliant material, TangoPlus, Stratasys, Minnesota, USA, to dampen the impact of the upper and lower hinges at maximum flexion and extension. Interchangeable bumpers of varying thickness allow the actuator to be configured with desired limits to knee flexion and extension. With no bumpers in place, the actuator's range of motion includes $112^{\circ}$ flexion and $-5^{\circ}$ hyperextension.

Connected to the bottom of the lower hinge is an adjustable pylon system. This system consists of a universal prosthetic pylon held by two tube clamps. Each tube clamp uses a single bolt to apply pressure around the circumference of the pylon, thus holding it in place. Due to this design, the distance between the two joints can be continuously adjusted for subjects with heights ranging from $1.52 \mathrm{~m}$ to $1.98 \mathrm{~m}$ (5' to 6' 6"), which can accommodate approximately 99.5 and 91.8 percent of all males and females, respectively [28]. The pylon can also be rotated by a prosthetist to properly align the abduction/adduction of the prosthetic leg's ankle actuator.

\section{F. Ankle Mechanical Structure}

Similar to the knee actuator, the ankle is designed with a single axis of rotation. Although the concept and capabilities of the two actuators are the same (i.e., torque and velocities), the physical layout of the ankle actuator, Figure 6, is different from that of the knee. At the knee, the axis of rotation of the motor and the joint output are coaxial. At the ankle, the motor axis of rotation is moved proximal to the body for two main reasons: users expend more metabolic energy wearing a mass that is more distal on the body [29], and overall actuator width would not allow the prosthetic foot to wear a cosmetic foot shell or shoe. With the motor and the transmission moved proximal to the body, a parallelogram 4-bar linkage mechanism was implemented to translate the torque from the output of the gearbox distal to the location of the anatomical ankle joint. Other powered prosthetic ankles have utilized linkage mechanisms to alter joint torque or align impact loads, however our linkage mechanism is only used to reduce metabolic energy expenditure and to satisfy size constraints [30], [31]. The ankle joint is mechanically constrained by hard stops located at approximately $\pm 45^{\circ}$. This provides ample rotation for a wide range of tasks, while preventing any harm to the user or system due to excessive ankle flexion. The 6-axis load cell is mounted directly below the ankle joint. Lastly, an off-the-shelf Ottobock LoRider prosthetic foot is attached to the bottom of the 6-axis load cell. The low profile of this foot in 
conjunction with the layout of this actuator leaves plenty of room for a cosmetic foot shell to be installed, allowing the user to wear most styles of shoes.

\section{Validation Experiments and Results}

This section presents several benchtop experiments that demonstrate the backdrivability, bandwidth, and position and impedance control capabilities of the prosthesis actuators. A supplemental video of these experiments is available for download.

\section{A. Backdrive Torque}

This test aims to quantify the backdrive torque of the actuators, i.e., the torque required at the output of an actuator to rotate the motor through its transmission. For this experiment, the ankle actuator was rigidly fixed to the benchtop setup with motion still being allowed at the ankle joint. A force was then applied with one finger to the toe of the foot (Figure 7). The applied force gradually increased until the joint moved. A total of nine trials of this experiment were conducted, three each with the ankle initially positioned at $-20^{\circ}, 0^{\circ}$, and $20^{\circ}$. For the case of $0^{\circ}$ and $20^{\circ}$, a downward force was applied to result in plantar flexion. For the case of $-20^{\circ}$, an upward force was applied to result in dorsiflexion.

Throughout this experiment torque data was collected from the 6-axis load cell. Torque maxima for each trial were extracted from the collected data and averaged for each initial starting position. These maxima occurred directly before the applied torque overcame static friction within the system. The magnitudes of the mean peak torque values were $3.41 \mathrm{Nm}$, $3.23 \mathrm{Nm}$, and $3.22 \mathrm{Nm}$ for the initial ankle position of $-20^{\circ}, 0^{\circ}$, and $20^{\circ}$, respectively. This confirms the actuator's ability to be backdriven with a low amount of torque.

\section{B. Free Swing}

A free-swinging knee has the benefit of simplifying control effort during swing phase, therefore leading to a more energy efficient system. Toward this end, we performed a simple experiment to show that the knee could be backdriven by the weight of the shank and foot alone, thus simulating the swing phase of gait. With the motors unpowered, four trials were performed in which the top of the knee was fixed to the benchtop setup, flexed between $65^{\circ}$ and $70^{\circ}$, and then released without a push. This experimental setup can be seen in Figure 8. Figure 9 shows the knee position for each of the four trials from the point of release until it reached the mechanical hard stop. With knee flexion peaking at approximately $70^{\circ}$ for level walking, it can be seen in Figure 9 that the knee does indeed exhibit free-swing capabilities since the knee repeatedly returns to zero after being released from a specified height.

It should be noted that the missing dynamics of walking not included in this experiment, such as hip moment and ankle push off, should decrease the free swing timing. In addition, the control strategy for walking, not included in this paper, could accelerate the free swinging knee motion. 


\section{Actuator Bandwidth}

Generally, real-world physical systems act as low-pass filters and hence attenuate high frequency inputs. In the case of actuators, and especially electric ones, the cut-off frequency of the system becomes an important factor in characterizing the speed by which the output (usually velocity) can be actively controlled through changing the input signal (usually commanded torque).

To determine the bandwidth of the designed motor/ transmission configuration of both joints, we tested the system on a benchtop setup by removing the mechanical output of the knee to ensure continuous rotation was not limited by the mechanical hard-stops. The experiment was done from very low frequency up to the point that the test was halted due to excessive shaking and vibrations, $0.05-12.75 \mathrm{~Hz}$. As the Bode plot in Figure 10 shows, the system has the standard characteristics of a first-order linear system with the phase starting at zero in low frequencies and ending at 90 degrees. The cut-off frequency was determined to be $2.3 \mathrm{~Hz}$. Noting that a frequency analysis of human gait shows that the highest frequency content of walking is in the range of $1-2 \mathrm{~Hz}$ [32], the actuator is expected to be completely capable of tracking the human-like joint trajectories with negligible errors. This will be demonstrated in the next set of experiments. Moreover, higher frequencies may be obtainable with aiding moments from the hip.

\section{Closed-Loop Position Control}

Many legged locomotion control paradigms, for instance those originating from Hybrid Zero Dynamics (HZD), rely on stiff and precise position control of the joints [10], [33]-[35]. To examine the actuators' position tracking capabilities, a proportional-derivative (PD) controller with a gravity compensation term was implemented for each actuator. For this experiment, both joints were assembled together and the complete leg was mounted onto the benchtop setup, as in Figure 8. The normative joint trajectories from [22] were tracked at frequencies of 0.5 (slow walking), 1.0 (fast walking), and $1.3 \mathrm{~Hz}$ (running) [32]. Figure 11 displays the tracking performance per joint for the frequencies mentioned.

Figure 11 displays tracking performance per joint for the increasing frequencies. For all three frequencies the ankle actuator is able to track the position with little error (max $0.27^{\circ}$, $0.45^{\circ}$, and $0.55^{\circ}$ for $0.5,1.0$, and $1.3 \mathrm{~Hz}$ respectively). Although the knee tracking errors are relatively small for 0.5 and $1 \mathrm{~Hz}\left(\max 1.04^{\circ}\right.$ and $6.42^{\circ}$ respectively), at $1.3 \mathrm{~Hz}$ the difference between desired and actual trajectories starts to become visible $\left(\max 13.17^{\circ}\right)$. This error is mainly due to phase lag between desired and measured trajectories. Neglecting this phase lag reduces the maximum knee tracking error to $2.05^{\circ}$ and $4.56^{\circ}$ for 1.0 and $1.3 \mathrm{~Hz}$, respectively. The higher error in the knee angle is due both to larger mass and inertia acting against the knee actuator as well as the larger range of motion and higher acceleration of the knee joint, compared to the ankle. It should also be noted that joint torque was saturated to $\pm 120 \mathrm{Nm}$ for safety during these tests, which could be relaxed in the future to reduce this error. Moreover, walking with the prosthesis will provide an aiding hip moment that could improve these results. Therefore, we expect the leg to be completely capable of supporting position-based control paradigms for the full range of walking speeds and some running speeds. 
Note that as the frequency increases, the first visible discrepancy between desired and actual trajectories appears at the knee flexion and extension immediately after the touchdown phase, which is the most difficult part of the cycle for the actuator to follow. In this region of gait, active position tracking is not strictly required because the function of the knee is to absorb energy during weight acceptance with the ground. In the next section, we show that different control paradigms (not based on position control) can take advantage of the actuator design to control this part of the gait more effectively, and as a result, can potentially achieve even higher frequencies.

\section{E. Open-Loop Impedance Control}

In the previous sets of experiments, we showed that the special design of the actuator and its high bandwidth makes it capable of supporting walking control paradigms designed based on precise joint position tracking. Here we show that the actuator design also works well for compliant walking control paradigms. This especially becomes important when one considers the most difficult part of the human joint trajectories to be mimicked by position control, namely the quick flexion and extension of the knee immediately after impact (Figure 11(f)). We know that in humans this happens due to natural compliance of the knee joint, rather than precisely following a prescribed position trajectory [26], [36]. This motivates us to test the ability of the designed actuator for specified impedance behaviors.

The most common way to perform joint impedance control is using joint torque feedback to produce the desired behavior. Note that for a fixed transmission ratio $n$ the general relationship between motor commanded torque $\tau_{m}$ and joint torque $\tau_{j}$ can be written as:

$$
\tau_{j}=\tau_{m}+n^{2} I_{m} \ddot{\theta}+n^{2} b_{m} \dot{\theta}+f(\theta, \dot{\theta}, t)
$$

where $I_{m}$ and $b_{m}$ are motor inertia and damping, respectively, $\theta$ is the joint angle, and $f$ contains nonlinear and time-dependent losses such as Coulomb friction, stiction and hysteresis. Note that $\tau_{m}=n k_{t} i_{m}$, where $k_{t}$ is the motor's torque constant and $i_{m}$ is its current, commanded to the driver. The torque feedback is necessary to decrease the effect of unmodeled dynamics $(f)$ and the usual uncertainty of inertia and damping parameters in Eq. 1. However, in this section we show that designing an actuator with minimal unmodeled dynamics essentially makes the impedance as part of the natural dynamics of the system, which gives the benefit of eliminating the torque sensor. This is especially important in a control problem such as walking where unexpected interactions with the environment (impacts) are always likely to occur, and the high noise and limited speed of closed-loop force control strongly motivates appropriate passive/natural dynamics.

In order for the actuator to work as a specified impedance, we simply set the position control PD gains, $K_{p}$ and $K_{d}$ equal to the desired spring and damper coefficients, respectively [37]. The position control is set to regulate a fixed angle (zero) as a person tries to move the ankle joint by hand. Then the commanded torque will be $\tau_{m}=-K_{p} \theta-K_{d} \theta$, which is the same as a spring-damper system. The six-axis load cell is used at the joint to measure the torque applied by the person (which is the same as joint torque), and compare it to the commanded torque $^{1}$. In an ideal case, these two torques will be equal, i.e., $\tau_{j}=\tau_{m}$. 
Figure 12 depicts the resulting ankle torques of four different experimental cases. The first and second case, Figure 12(a-b), show pure stiffness and pure damping tests, respectively, whereas cases three and four, Figure 12(c-d), depict a combined stiffness-damping control. As the figures show, there is a very good agreement between measured joint torque and commanded motor torque in the first three cases, proving that the effect of unmodeled dynamics is negligible for torques over $\sim 10-20 \mathrm{Nm}$. Note that this is much smaller than joint torques during stance phase [22], making the actuator completely suitable for any kind of compliant control during stance. To investigate the limitation of the control for low torques (where the effect of unmodeled dynamics becomes considerable), we applied small torque to the end-effector which shows a noticeable difference between the two torques for amplitudes less than $\sim 5 \mathrm{Nm}$ (Figure 12(d)). Interestingly, the difference between torques is around the value obtained for the backdriving torque as presented before $(\sim 3 \mathrm{Nm})$.

\section{Conclusion}

This paper presented the design and experimental validation for a powered prosthetic leg with high torque density actuators. The system implements high torque motors coupled with low reduction transmissions. Low mechanical impedance is an inherent feature of the actuator's design, resulting in low backdrive torques to move the motor.

Experimental tests were performed to determine certain characteristics of the actuators. Due to the low mechanical impedance, the knee actuator exhibits free-swing under the weight of the leg alone. Bandwidth tests revealed the actuators' ability to achieve frequencies common for fast gait patterns. Closed-loop position control implemented on the leg was shown to be effective, resulting in negligible error for frequencies up to $1.3 \mathrm{~Hz}$. Open-loop impedance control tests proved that the effect of unmodeled dynamics is negligible for torques over 10$20 \mathrm{Nm}$, making the actuator suitable for any kind of compliant control during stance phase of gait. The low actuator impedance and accurate impedance control make it possible to command and control the torque of the system without any torque feedback, thus allowing the removal of torque sensors from the system's design.

Future work for this powered prosthetic leg involves consolidating the computer, power supply, and other electronics on-board to create a self-contained prosthesis. Additional development of this prosthesis may leverage the backdriveability of the actuators to implement power regeneration when negative work is being done on the leg. Such a system would aim to safely and efficiently reharvest power during these periods. Upon becoming untethered from the benchtop, this prosthetic leg will be used as a platform for control prototyping and used in amputee experiments. Lastly, in an attempt to reduce weight of the self contained unit, we will further optimize component design and material selection.

\section{Supplementary Material}

Refer to Web version on PubMed Central for supplementary material.

\footnotetext{
${ }^{1}$ Note that in these experiments the load cell is merely used for diagnostics and not for any kind of feedback control.
} 


\section{Acknowledgments}

This work was supported by the National Institute of Child Health \& Human Development of the NIH under Award Number DP2HD080349. This work was also supported by NSF Award 1637704. The content is solely the responsibility of the authors and does not necessarily represent the official views of the NIH or the NSF. Robert D. Gregg, IV, Ph.D., holds a Career Award at the Scientific Interface from the Burroughs Wellcome Fund.

The authors thank Yonatan Chatzinoff and David Merz for assisting the mechanical design and Lukas Zekarias for assisting the programming.

\section{References}

1. Miller W, Deathe A, Speechley M, Koval J. The influence of falling, fear of falling, and balance confidence on prosthetic mobility and social activity among individuals with a lower extremity amputation. Arch. Phys. Med. Rehab. 82(9):1238-1244.2001;

2. Gailey RS, Wenger MA, Raya M, Kirk N, Erbs K, Spyropoulos P, Nash MS. Energy expenditure of trans-tibial amputees during ambulation at self-selected pace. Prosthetics Orthotics Int. 18(2):84.1994;

3. Kaufman KR, Frittoli S, Frigo CA. Gait asymmetry of transfemoral amputees using mechanical and microprocessor-controlled prosthetic knees. Clinical Biomechanics. 27(5):460-465.2012; [PubMed: 22221344]

4. Lambrecht, BG, Kazerooni, H. Robotics and Automation, 2009. ICRA'09. IEEE International Conference on. IEEE; 2009. Design of a semi-active knee prosthesis; 639-645.

5. Berry D. Microprocessor prosthetic knees. Physical Medicine and Rehabilitation Clinics. 17(1):91113.2006; [PubMed: 16517347]

6. Pieringer, DS; Grimmer, M; Russold, MF; Riener, R. Review of the actuators of active knee prostheses and their target design outputs for activities of daily living; IEEE Int. Conf. Rehabilitation Robotics; 2017. 1246-1253.

7. Tucker MR, Olivier J, Pagel A, Bleuler H, Bouri M, Lambercy O, del R Millàn J, Riener R, Vallery H, Gassert R. Control strategies for active lower extremity prosthetics and orthotics: a review. J. Neuroeng. Rehabil. 12(1)2015;

8. Fu, A, Fu, C, Wang, K, Zhao, D, Chen, X, Chen, K. Robotics and Biomimetics (ROBIO), 2013 IEEE International Conference on. IEEE; 2013. The key parameter selection in design of an active electrical transfemoral prosthesis; 1716-1721.

9. Yang U-J, Kim J-Y. Mechanical design of powered prosthetic leg and walking pattern generation based on motion capture data. Advanced Robotics. 29(16):1061-1079.2015;

10. Quintero, D; Villarreal, DJ; Gregg, RD. Preliminary experimental results of a unified controller for a powered knee-ankle prosthetic leg over various walking speeds; IEEE Int. Conf. Intelligent Robots Systems; 2016. 5427-5433.

11. Quintero D, Villarreal DJ, Lambert D, Kapp S, Gregg RD. Continuous-phase control of a powered knee-ankle prosthesis: Amputee experiment sacross speeds and inclines. IEEE Transactions on Robotics. 2018

12. Pillai, MV, Kazerooni, H, Hurwich, A. Robotics and Automation (ICRA), 2011 IEEE International Conference on. IEEE; 2011. Design of a semi-active knee-ankle prosthesis; 5293-5300.

13. Lawson BE, Mitchell J, Truex D, Shultz A, Ledoux E, Goldfarb M. A robotic leg prosthesis: Design, control, and implementation. IEEE Robotics \& Automation Magazine. 21(4):70-81.2014;

14. Au SK, Herr H. Powered ankle-foot prosthesis. IEEE Robot. Automat. Mag. 15(3):52-59.2008;

15. Sup F, Varol HA, Mitchell J, Withrow TJ, Goldfarb M. Preliminary evaluations of a self-contained anthropomorphic transfemoral prosthesis. IEEE/ASME Transactions on Mechatronics. 14(6):667676.2009; [PubMed: 20054424]

16. Sup F, Bohara A, Goldfarb M. Design and control of a powered transfemoral prosthesis. Int. J. Robot. Res. 27(2):263-273.2008;

17. Seok S, Wang A, Chuah MYM, Hyun DJ, Lee J, Otten DM, Lang JH, Kim S. Design principles for energy-efficient legged locomotion and implementation on the mit cheetah robot. IEEE/ASME Transactions on Mechatronics. 20(3):1117-1129.2015; 
18. Hubicki C, Grimes J, Jones M, Renjewski D, Spröwitz A, Abate A, Hurst J. Atrias: Design and validation of a tether-free 3d-capable spring-mass bipedal robot. The International Journal of Robotics Research. 35(12):1497-1521.2016;

19. Kenneally G, De A, Koditschek DE. Design principles for a family of direct-drive legged robots. IEEE Robotics and Automation Letters. 1(2):900-907.2016;

20. Zhu, H; Doan, J; Stence, C; Lv, G; Elery, T; Gregg, RD. Design and validation of a torque dense, highly backdrivable powered knee-ankle orthosis; IEEE Int. Conf. Robotics Autom; 2017. 504510.

21. Levai Z. Structure and analysis of planetary gear trains. Journal of mechanisms. 3(3):131148.1968;

22. Winter, DA. Biomechanics and Motor Control of Human Movement. 2. New York, NY: Wiley; 2009.

23. Embry, KR; Villarreal, DJ; Gregg, RD. A unified parameterization of human gait across ambulation modes; IEEE Eng. Med. Bio. Conf; 2016.

24. Gupta V, Deb A. Analysis of variable gear system on energy consumption in electric vehicle using simulation tool. IJSSST. 13(2):7-11.2012;

25. Ding, Y; Park, HW. Design and experimental implementation of a quasi-direct-drive leg for optimized jumping; 2017 IEEE/RSJ International Conference on Intelligent Robots and Systems (IROS); 2017. 300-305.

26. Smidt GL. Biomechanical analysis of knee flexion and extension. Journal of biomechanics. 6(1):79IN1381-8092.1973; [PubMed: 4693870]

27. Pfeifer, S, Riener, R, Vallery, H. Biomedical Robotics and Biomechatronics (BioRob), 2012 4th IEEE RAS \& EMBS International Conference on. IEEE; 2012. An actuated transfemoral prosthesis with optimized polycentric knee joint; 1807-1812.

28. Census.gov. Table 205. cumulative percent distribution of population by height and sex: 2007 to 2008. 2011. [Online]. Available: https://www2.census.gov/library/publications/2010/compendia/ statab/130ed/tables/11s0205.pdf

29. Browning RC, Modica JR, Kram R, Goswami A. The effects of adding mass to the legs on the energetics and biomechanics of walking. Medicine \& Science in Sports \& Exercise. 39(3):515525.2007; [PubMed: 17473778]

30. Cempini, M; Hargrove, LJ; Lenzi, T. Design, development, and bench-top testing of a powered polycentric ankle prosthesis; 2017 IEEE/RSJ International Conference on Intelligent Robots and Systems (IROS); 2017. 1064-1069.

31. LaPre, AK, Sup, F. Rehabilitation Robotics (ICORR), 2013 IEEE International Conference on. IEEE; 2013. Redefining prosthetic ankle mechanics: Nonanthropomorphic ankle design; 1-5.

32. Satkunskienė D, Grigas V, Eidukynas V, Domeika A. Acceleration based evaluation of the human walking and running parameters. Journal of Vibroengineering. 11(1):506-510.2009;

33. Grizzle JW, Abba G, Plestan F. Asymptotically stable walking for biped robots: Analysis via systems with impulse effects. IEEE Transactions on automatic control. 46(1):51-64.2001;

34. Martin A, Gregg RD. Stable, robust hybrid zero dynamics control of powered lower-limb prostheses. IEEE Trans. Automatic Control. 62(8):3930-3942.2017; [PubMed: 29276305]

35. Westervelt, E, Grizzle, J, Chevallereau, C, Choi, J, Morris, B. Feedback Control of Dynamic Bipedal Robot Locomotion. New York, NY: CRC Press; 2007.

36. Shamaei K, Sawicki GS, Dollar AM. Estimation of quasi-stiffness of the human knee in the stance phase of walking. PloS one. 8(3):e59993.2013; [PubMed: 23533662]

37. Hogan N. Impedance control: An approach to manipulation. Journal of dynamic systems, measurement, and control. 107(1):8-16.1985; 


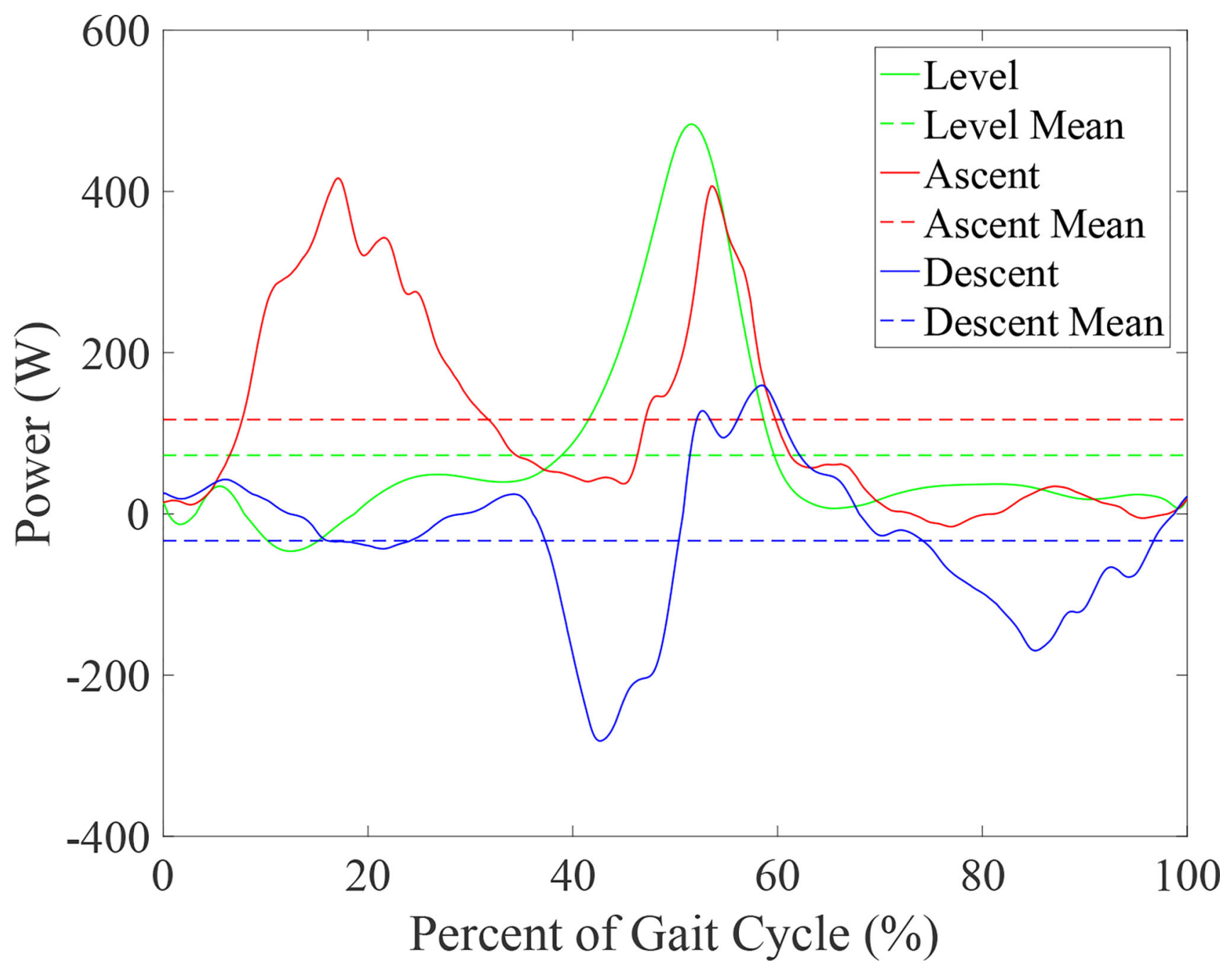

Fig. 1.

Power requirements of the prosthetic leg system. This includes power required for both motors simultaneously and the electronics system. 

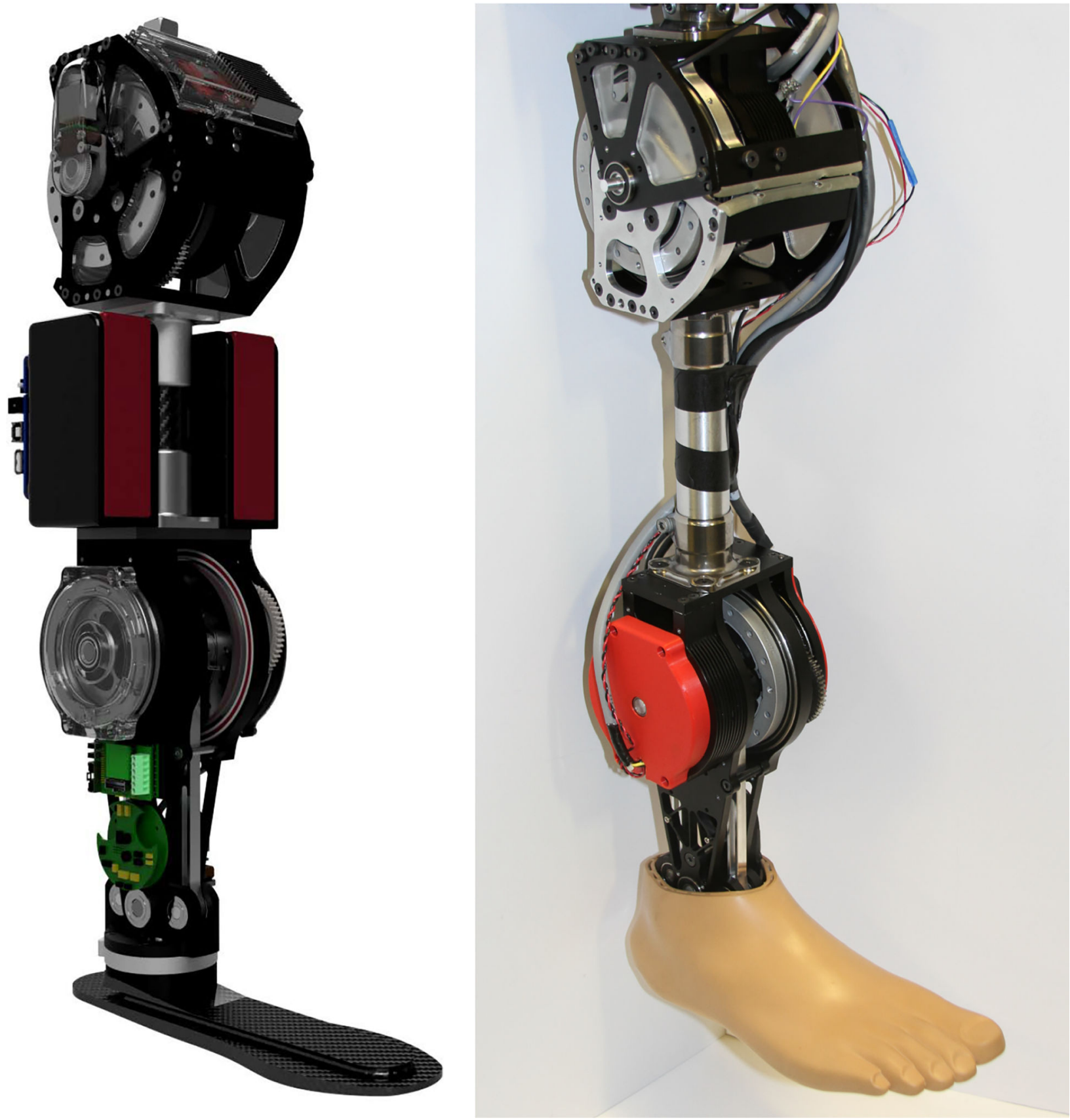

Fig. 2.

Final assembly of the prosthetic leg. The image on the left displays a CAD rendering, and the image on the right displays the physical system after assembly. Note that for simplicity, certain components of the electronics system shown in the rendering are located on the benchtop for the experiments presented in Section III. 

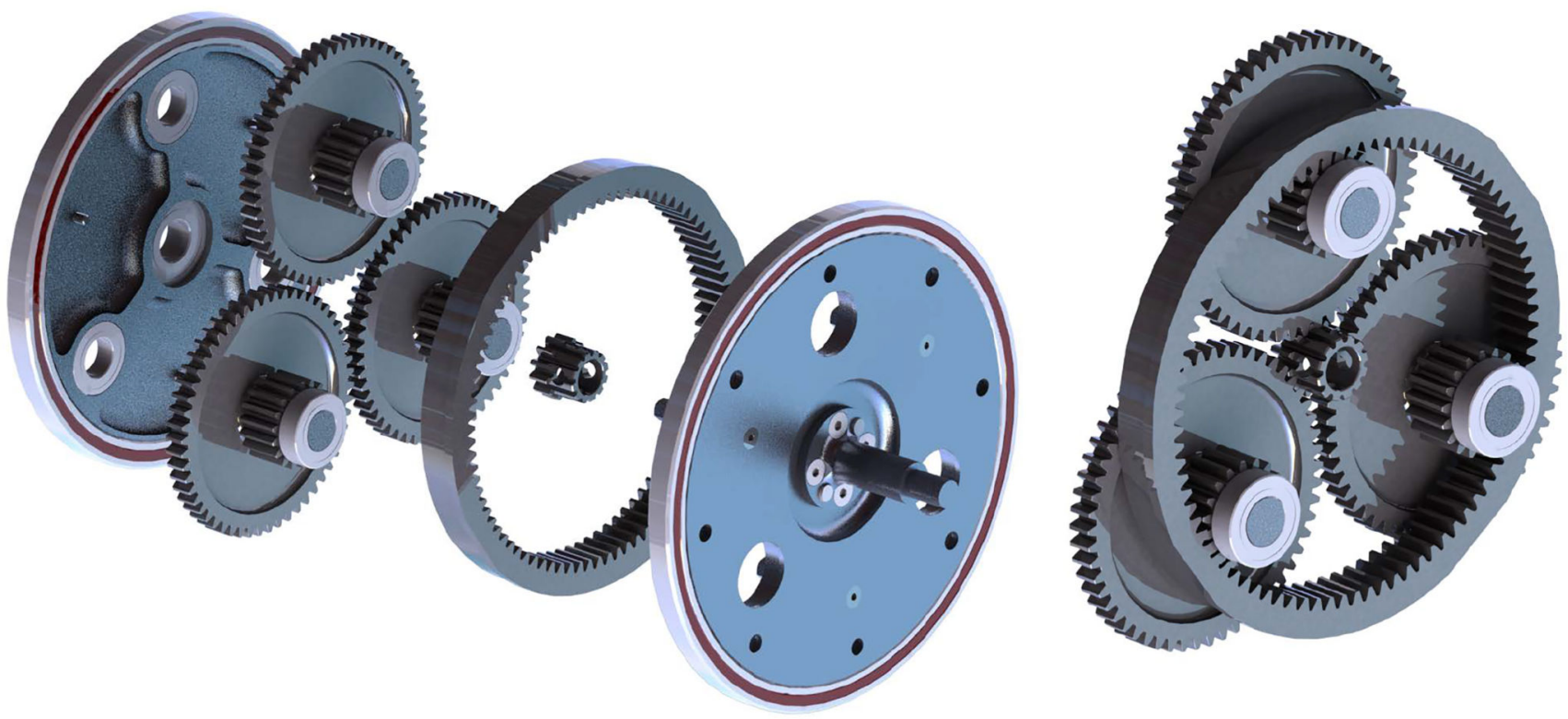

Fig. 3.

CAD model of the planetary gear transmission. The image on the left illustrates an exploded view of the entire transmission (including planet carriers), while the right demonstrates the gear layout after assembly. 


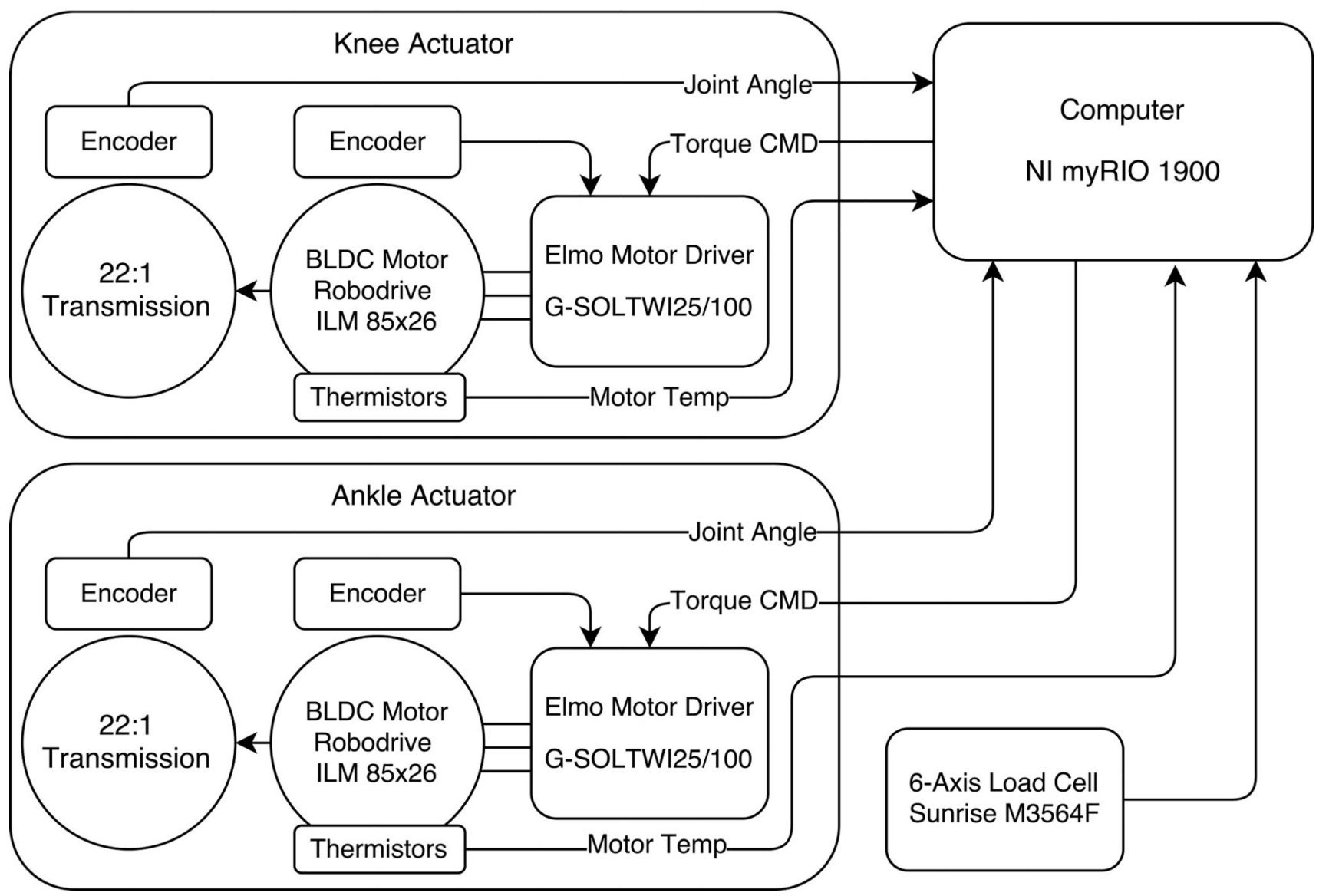

Fig. 4.

Block Diagram of Electrical System: The system's computer receives feedback related to the user's gait and sends torque commands. The two actuators control and drive the knee and ankle motors. 

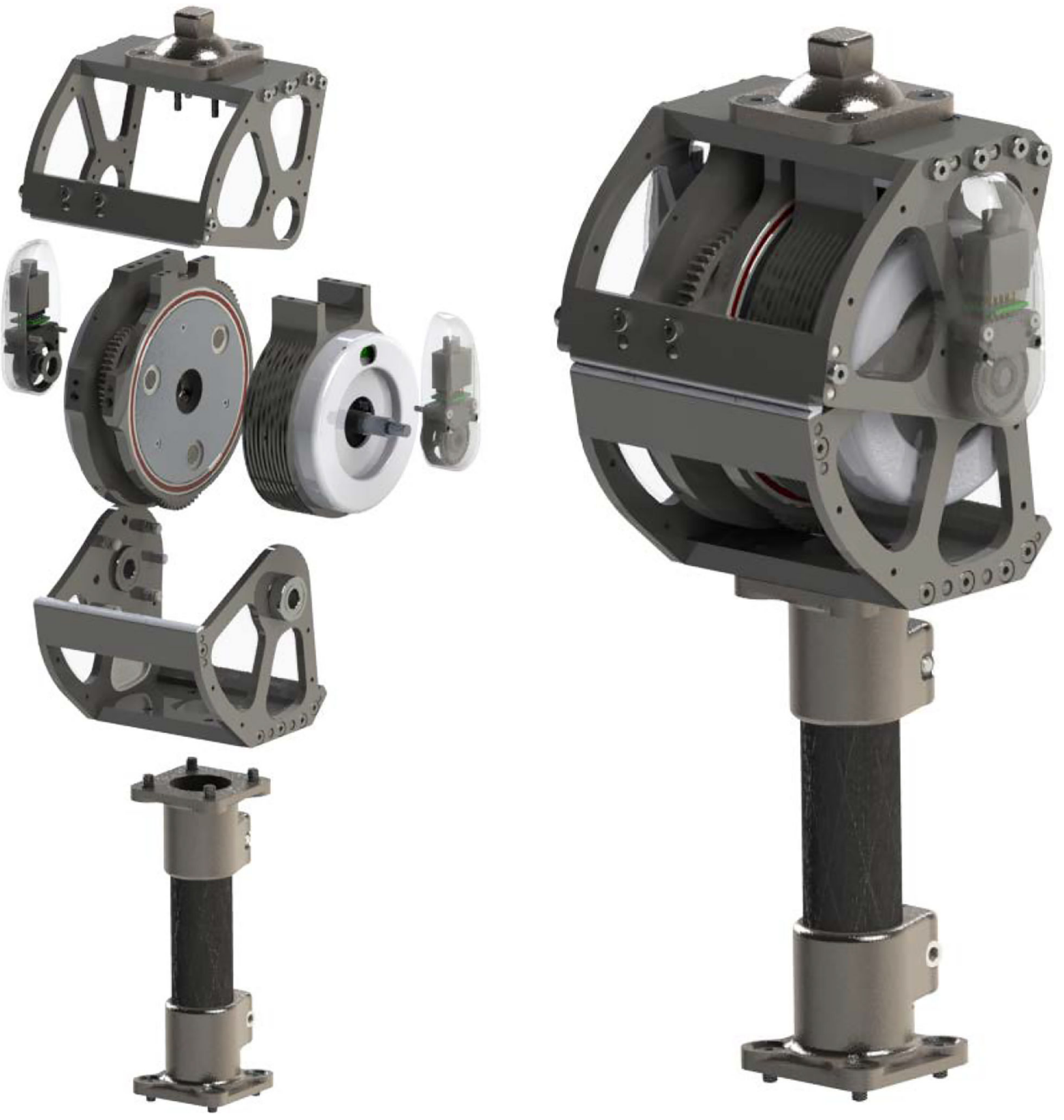

Fig. 5.

CAD design of the knee actuator. The exploded view on the left displays the components/ sub-assemblies of the knee actuator, such as the upper/lower hinges, encoders, transmission, motor, and pylon. The image on the right presents the assembled knee actuator. The pyramid adapter on top connects to the user's socket, and the length-adjustable pylon on bottom connects to the ankle actuator module. 

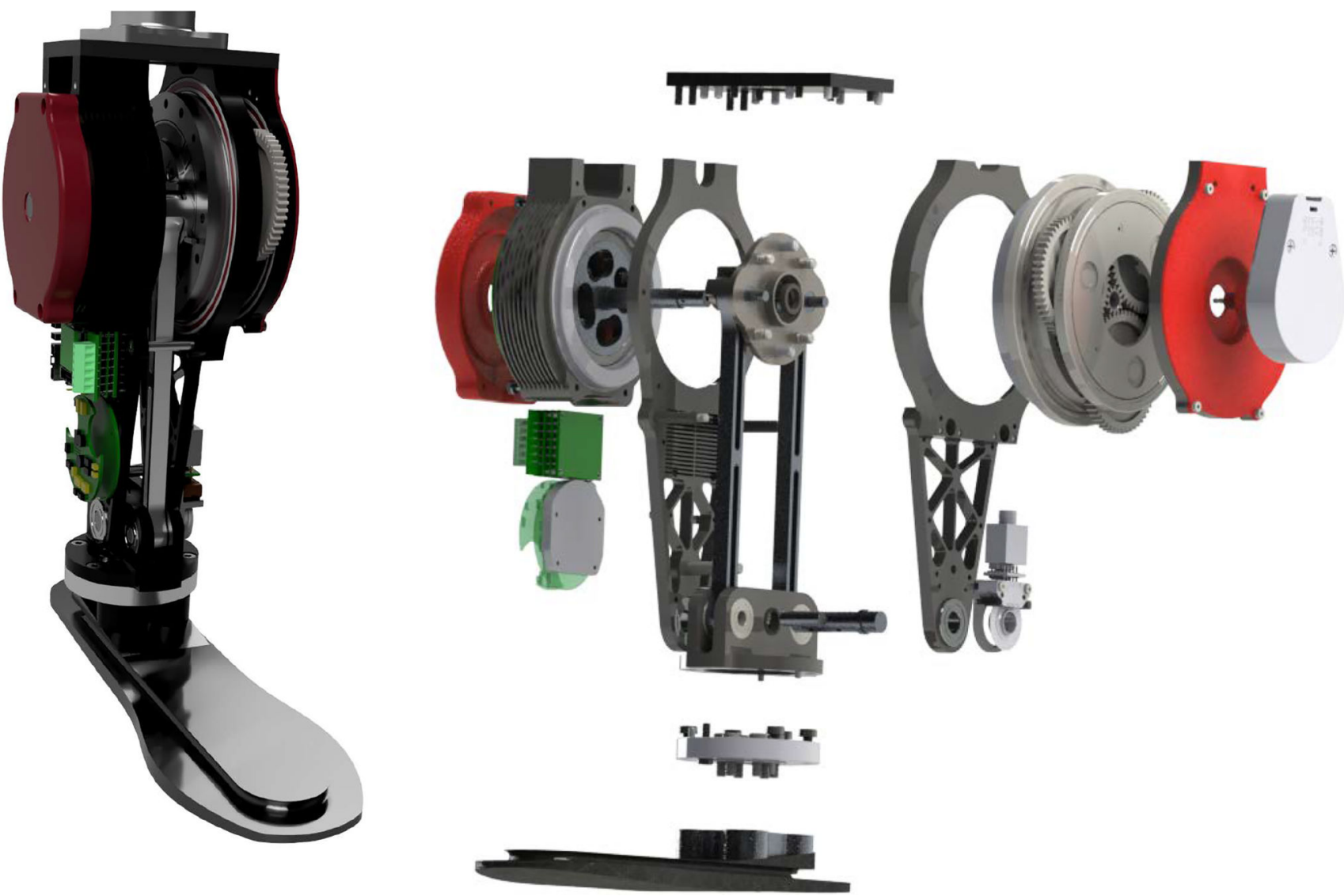

Fig. 6.

CAD design of the ankle actuator. The image on the left presents the assembled ankle actuator. The exploded view on the right displays the components/sub-assemblies of the ankle actuator, such as the motor, structure, 4-bar linkage, transmission, electronics, and foot. 


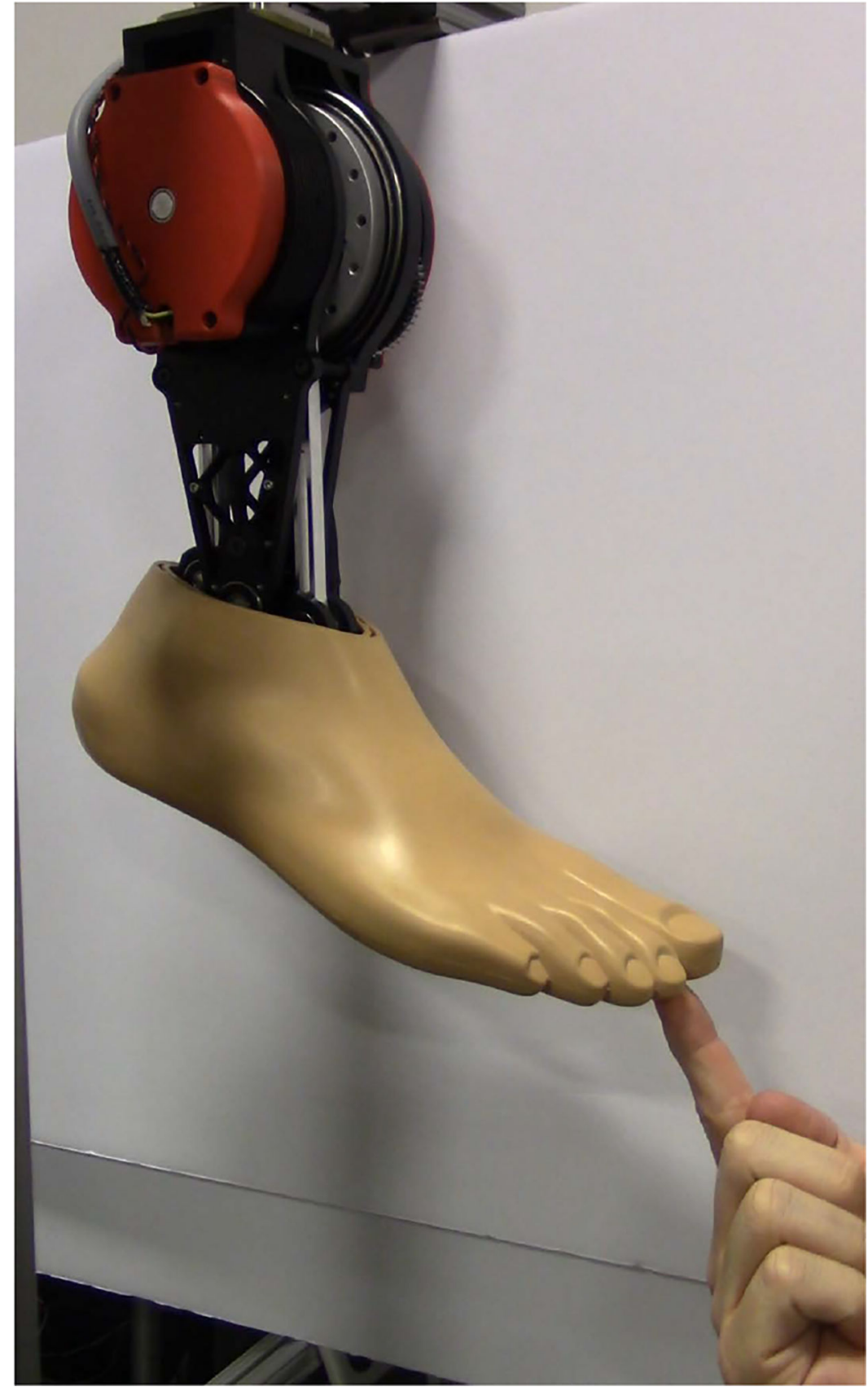

Fig. 7.

Experimental setup for backdrive torque test. 

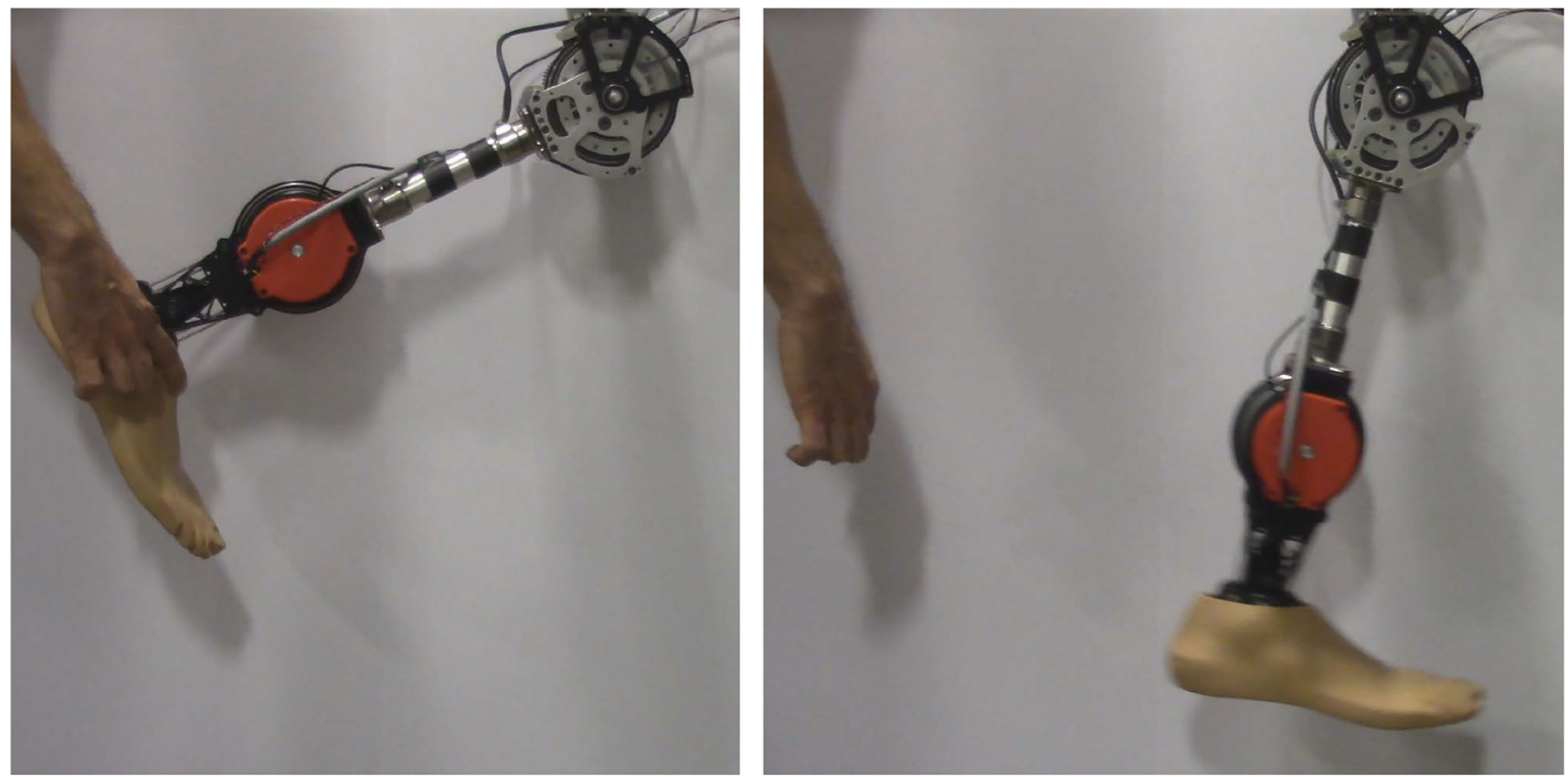

Fig. 8.

Experimental setup for free swing test. The photo on the left shows the unpowered leg when the knee is held in flexion. The photo on the right shows the shank of the leg in motion after being released. 


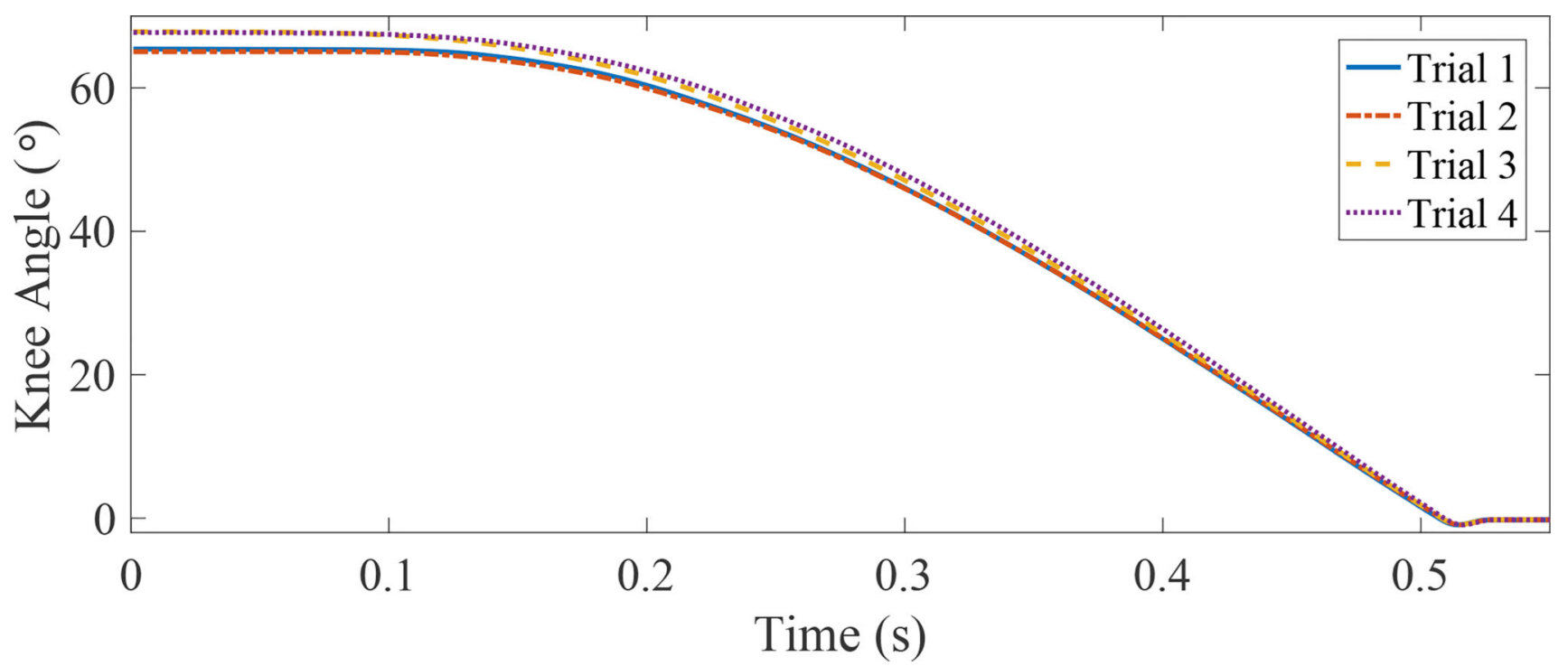

Fig. 9.

Recorded position of the knee as it returns to zero following release from an initial offset. 

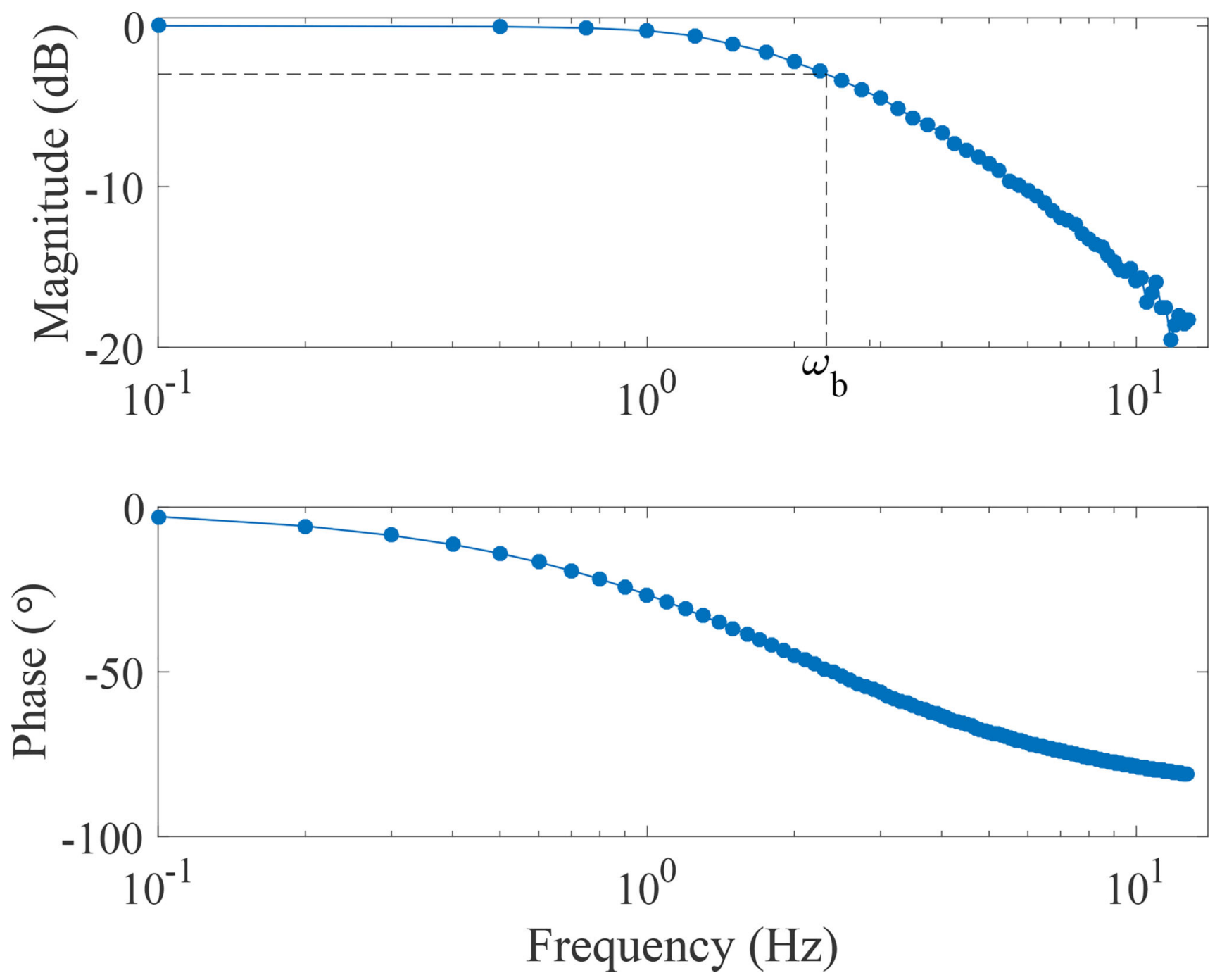

Fig. 10.

Bode plots for open-loop velocity bandwidth tests. The top figure displays the magnitude and cutoff frequency $\omega_{b}$ of $2.32 \mathrm{~Hz}$, where the bottom presents the phase shift as frequency increases. 
Ankle

(a)

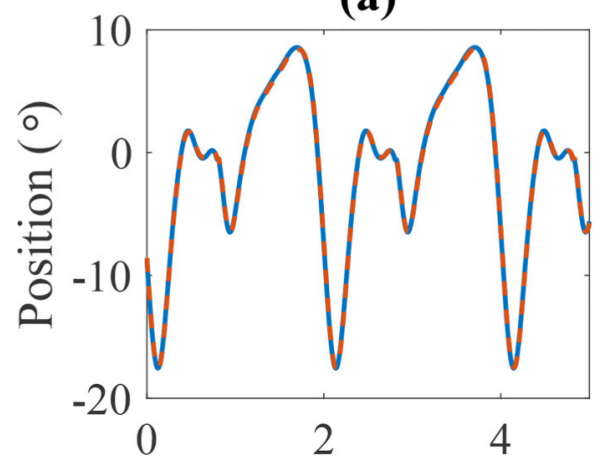

(c)

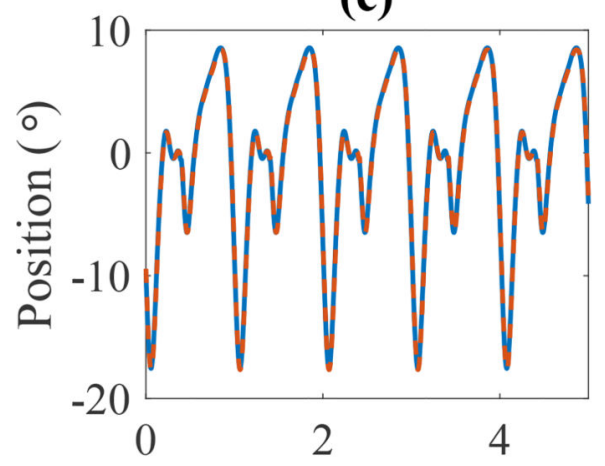

(e)

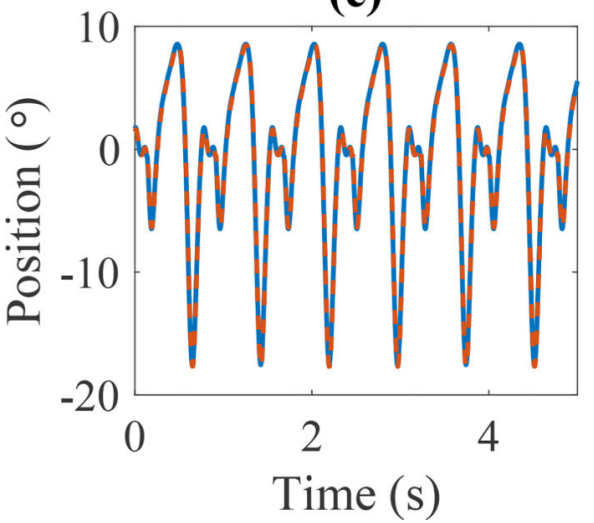

Knee

(b)

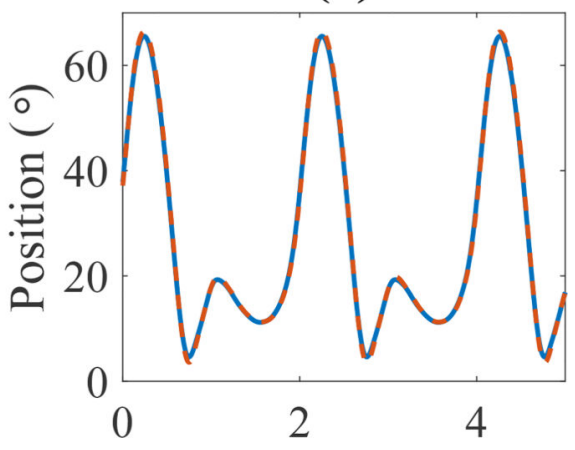

(d)

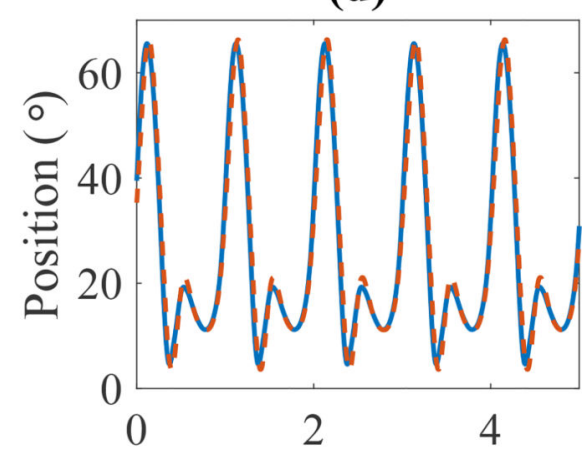

(f)

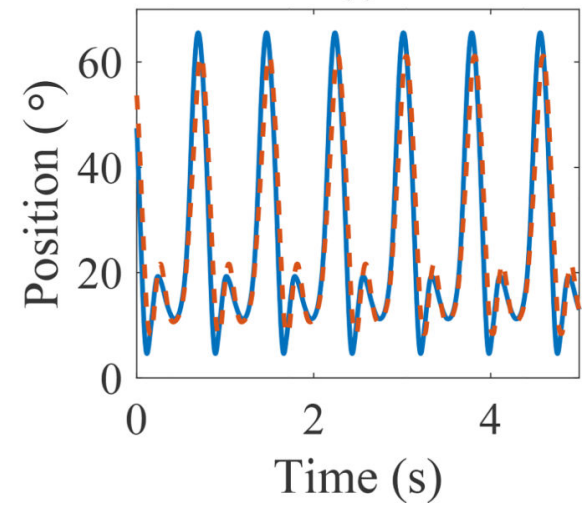

Fig. 11.

Position tracking of normative gait trajectories at various frequencies. Solid blue and dotted red lines denote the desired and measured position, respectively. Plots a), c), and e) present ankle tracking at $0.5,1.0$, and $1.3 \mathrm{~Hz}$ respectively. Plots b), d), and f) present knee tracking at $0.5,1.0$, and $1.3 \mathrm{~Hz}$ respectively. 
(a)

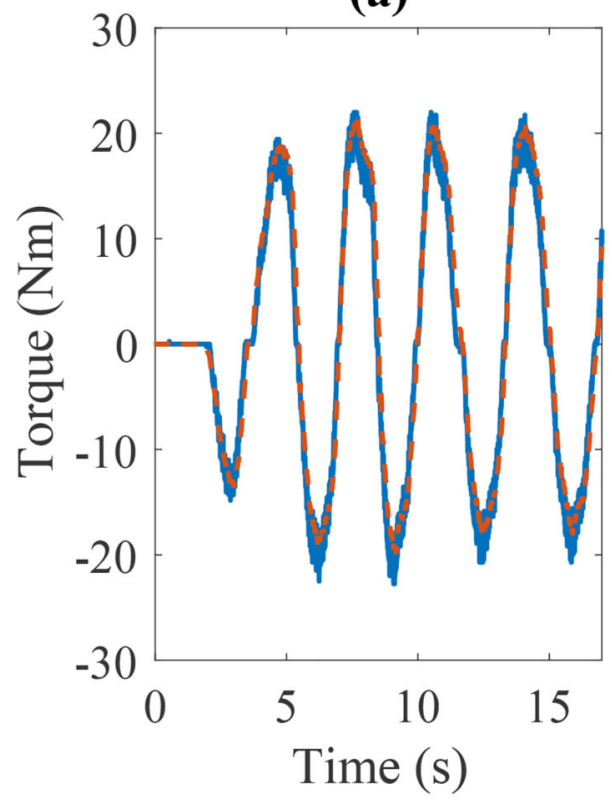

(c)

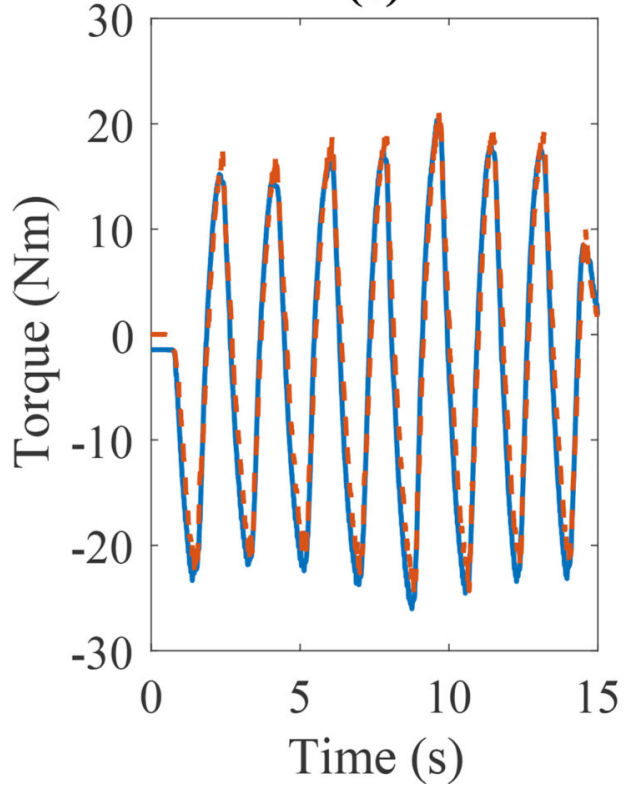

(b)

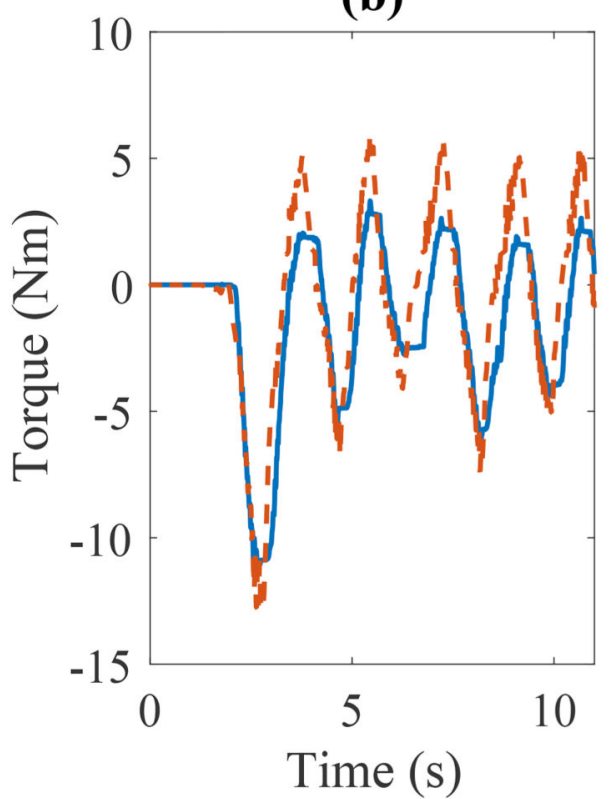

(d)

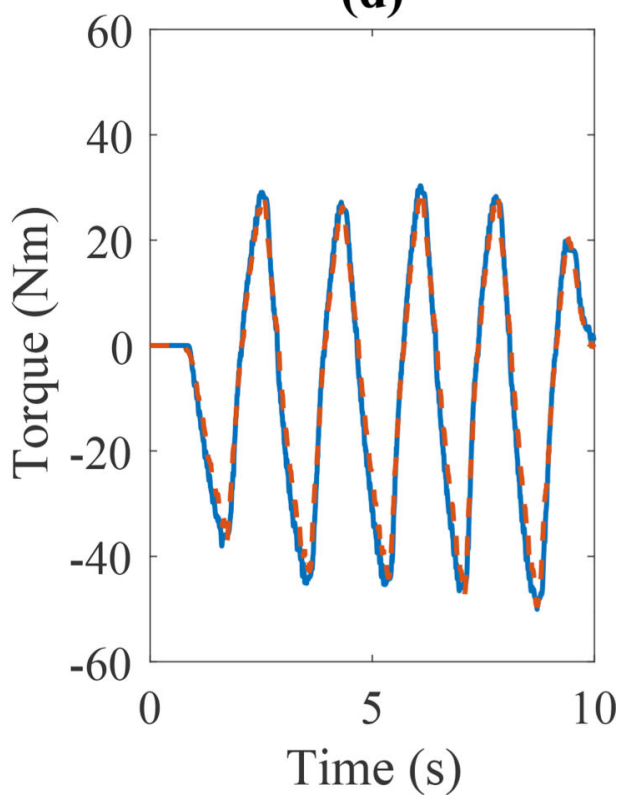

Fig. 12.

Open-loop impedance of the ankle joint with various $K_{p}$ and $K_{d}$ gains. Solid blue and dotted red lines correspond to commanded and measured torque respectively. Reduced PD gains used are: a) $K_{p}=0$ and $K_{d}=0.5$, b) $K_{p}=0.8$ and $K_{d}=0.05$, c) $K_{p}=0.8$ and $K_{C}=0.05$, and d) $K_{p}=3$ and $K_{C}=0.15$. 


\section{TABLE I}

Combined Knee and ankle power requirements

\begin{tabular}{|c|c|c|}
\hline & Ankle Requirements & Knee Requirements \\
\hline Torque & $140 \mathrm{Nm}$ & $115 \mathrm{Nm}$ \\
\hline Velocity & $360^{\circ} / \mathrm{s}$ & $330^{\circ} \mathrm{s}$ \\
\hline Position & $-28^{\circ}$ to $20^{\circ}$ & $0^{\circ}$ to $105^{\circ}$ \\
\hline Power & $307 \mathrm{~W}$ & $225 \mathrm{~W}$ \\
\hline
\end{tabular}

